ADP-09-16/T694

JLAB-THY-09-1097

\title{
Understanding the proton's spin structure
}

\author{
F. Myhrer ${ }^{a}$ and A.W. Thomas ${ }^{b, c}$ \\ ${ }^{a}$ Department of Physics and Astronomy, University of South \\ Carolina, Columbia, SC 29208 \\ b Jefferson Lab, 12000 Jefferson Ave., \\ Newport News, VA 23606 \\ ${ }^{c}$ Centre for the Subatomic Structure of Matter and School of \\ Chemistry and Physics, \\ The University of Adelaide, Adelaide SA 5005, Australia
}

(October 29, 2018)

\begin{abstract}
We discuss the tremendous progress that has been towards an understanding of how the spin of the proton is distributed on its quark and gluon constituents. This is a problem that began in earnest twenty years ago with the discovery of the proton "spin crisis" by the European Muon Collaboration. The discoveries prompted by that original work have given us unprecedented insight into the amount of spin carried by polarized gluons and the orbital angular momentum of the quarks.
\end{abstract}




\section{Introduction}

The spin structure of the proton, or how the spins of the quarks and polarized gluons and their orbital angular momenta make up the total angular momentum $1 / 2$, has been a puzzle since the discovery by the European Muon Collaboration (EMC) that the quarks appeared to contribute very little to the proton spin [1]. This surprising experimental result appears to be in contrast to the apparently successful, naive quark model descriptions of the structure of the proton, such as the proton's charge radius, magnetic moment and axial charge. The explanation of these nucleon structure observables has its basis in QCD, the theory of strong interaction, which is invariant under chiral transformations provided that the $u$ and $d$ quarks are massless. Furthermore, chiral perturbation theory (ChPT), an effective low-energy theory of QCD, assumes that the Goldstone Boson of the theory is the effective pion-field, which more properly should be considered a coherent state of quarks and anti-quarks. We know that chiral symmetry is explicitly broken by non-zero quark masses and according to ChPT the pion mass is generated by the small $u$ and $d$ quark masses $m_{q}$, leading to the relation $m_{\pi}^{2} \propto m_{q}$.

These ideas have established that the low-momentum structure of the nucleon consists of a core of three confined, interacting valence quarks (the "bare" nucleon) plus a pion cloud which interacts with the valence quarks of the quark core in a manner dictated by chiral symmetry. This description of the nucleon was however not capable of reproducing the initial EMC measurement [1] of the very small quark spin contribution to the proton spin. As will be presented in this topical review the above description of the proton's structure does explain the updated measurements of the quark spin contribution.

The proton spin structure is experimentally explored in deep inelastic scattering (DIS) and polarized proton proton reactions where the four-momentum transfer squared, $Q^{2}$, is large compared to the hadronic scale $\sim 1(\mathrm{GeV} / \mathrm{c})^{2}$. At these large values of $Q^{2}$ it appears natural to use simple parton model considerations to analyze the experimentally measured structure functions which are functions of $Q^{2}$ and the Bjorken variable $x$. The measured dependence of the structure functions on $Q^{2}$ is consistent with the expected perturbative QCD behavior down to surprisingly low $Q^{2}$ values. However, we know that even at high $Q^{2}$ the three confined valence quarks in the proton retain their transverse degrees of freedom, such as the transverse momentum $k_{\perp}$, and these transverse degrees of freedom can have a non-negligible influ- 
ence on the interpretation of the experimental spin-dependent data. We will elaborate on this topic in the fifth section of this review.

In the next section we briefly present experimental data which are relevant to the proton spin structure. The focus will be on recent experimental results which appear to indicate that polarized gluons contribute little to the proton spin. In the third section we concentrate on well-known non-perturbative aspects of QCD which successfully explain the recently determined first moment value, $\Sigma$, of the measured proton spin structure function $g_{1}(x)$. In the fourth section we discuss the recent lattice evaluations of the $u$ - and $d$ - quark angular momenta. Finally, before summarizing this review, we will discuss the transverse momentum distributions of the quarks which affect the spin distributions of the proton. This fifth section also include a brief discussion of the transverse structure functions measurements necessary in order to verify experimentally the consequences implied by our explanation of $\Sigma$, namely that the quark and antiquark orbital angular momentum contributions to the proton spin are sizable.

\section{A summary of the experimental data}

The EMC experimental result that $\Sigma$ is small [1], the so-called proton "spin crisis", generated a tremendous effort in order to map out the quark and gluon distributions of energy, momentum, spin and angular momentum of the proton. The experimental effort at CERN [1, 2, 3, 4], DESY [5], JLab [6], RHIC [7, 8] and SLAC [9] over the past two decades has been very impressive and today several crucial pieces of information have been established. The longitudinal spin structure function $g_{1}\left(x, Q^{2}\right)$ has been measured over a range of values of $Q^{2}$ and for $x$ down to $10^{-4}$ - although at the lower x values the COMPASS data [4] on deuterium is below $Q^{2}=1 \mathrm{GeV}^{2}$.

The quark spin contribution, $\Sigma$, measures the quark (and antiquark) helicity along the longitudinal proton spin minus the quark (and antiquark) helicity antiparallel to the proton spin. At very large $Q^{2}$ values the integral of the proton spin structure function, $g_{1}\left(x, Q^{2}\right)$, can be written as

$$
\Gamma^{p}\left(Q^{2}\right)=\int_{0}^{1} \mathrm{~d} x g_{1}^{p}\left(x, Q^{2}\right)=\frac{c_{1}\left(Q^{2}\right)}{12}\left[g_{A}^{(3)}+\frac{1}{3} g_{A}^{(8)}\right]+\frac{c_{2}\left(Q^{2}\right)}{9} \Sigma
$$

The low-energy nucleon axial coupling constant, $g_{A}^{(3)}=g_{A}$ is very well known from neutron $\beta$-decay, while $g_{A}^{(8)}$ has been determined from semi-leptonic hyperon decay with an error that is often quoted as $5 \%$ but may be as large 
as $20 \%$ [10]. The flavor-singlet axial-current matrix element, $\Sigma$, is the focus of this review. The non-singlet and singlet "radiative" coefficients, $c_{1}\left(Q^{2}\right)$ and $c_{2}\left(Q^{2}\right)$ respectively, have been evaluated in QCD perturbation theory at the three-loop level [11, 12]. As $Q^{2} \rightarrow \infty$ both coefficients approach 1 .

A summary of the status and recent experimental results on the spin structure of the nucleon can be found in Ref. [13]. Additionally, we refer to the recent work of Bass [14 who stresses that the isovector and isoscalar components of the measured spin structure function $g_{1}(x)$ behave very differently at small $x$-values [5], and he presents possible reasons for these different behaviors.

Unlike the early EMC result that the quark spin contribution $\Sigma$ was consistent with zero, $14 \pm 9 \pm 21 \%$ [1], today we know that the sum of the helicities of the quarks in the proton is about a third of the total spin [3, 4, 5],

$$
\Sigma=0.33 \pm 0.03 \text { (stat.) } \pm 0.05 \text { (syst.) } .
$$

This result is small compared to $\Sigma_{\text {quark }} \sim 0.67$, the expected value obtained when one considers the relativistic motion of the confined valence quarks, which we will discuss in the next section.

The initial, extremely low EMC value of $\Sigma$ raised the exciting possibility that the proton could contain a substantial quantity of polarized gluons, which can contribute to $g_{1}^{p}(x)$ through the axial $U(1)$ anomaly [15, 16, 17, 18, 19, 20]. The essential point is that the flavor-singlet axial current is not conserved because of this anomaly. To account for the effect of the anomaly the matrix element of the axial charge $\Sigma$ in Eq.(1) could be written as (see the review by Bass [21]):

$$
\Sigma=\Sigma_{\text {quark }}-\frac{N_{f} \alpha_{s}\left(Q^{2}\right)}{2 \pi} \Delta G\left(Q^{2}\right)
$$

Here $\Sigma_{\text {quark }}$ is the quark model prediction and the second term is the contribution of polarized gluons arising from the axial anomaly. In the limit $Q^{2} \rightarrow \infty$ the product $\alpha_{s}\left(Q^{2}\right) \Delta G\left(Q^{2}\right)$ has a non-zero value - see e.g. Refs. [15, 16, 17]. The question pursued by several experimental groups is whether the polarized gluon content of the proton, $\Delta G\left(Q^{2}\right)$, is large enough to explain the measured value of $\Sigma$ reported in Eq.(2).

If we ascribe the unexpectedly small observed $\Sigma$ value in Eq.(2) as being entirely caused by the polarized gluons in the proton, we can estimate the value of $\Delta G\left(Q^{2}\right)$ expected from experiments. At a scale of $Q^{2} \simeq 3(\mathrm{GeV} / \mathrm{c})^{2}$ 
we know $\alpha_{s}\left(Q^{2}\right) \simeq 0.3$. When we include only the relativistic corrections due to the confined quarks' motions in the proton giving $\Sigma_{\text {quark }} \simeq 0.67$, we obtain from Eqs.(2) and (3) with three flavors a value for $\Delta G\left(Q^{2}\right) \simeq 2.4$. The recent experimental data however indicate that the polarization of the gluons is much smaller, typically only one tenth of this.

At the SPIN2008 conference Rondio summarized the status of the polarized gluon experiments 22 and concluded that most likely the gluon polarization is small. Most recently the COMPASS Collaboration used an idea proposed in Ref. [17] to measure the polarized gluons in the proton. They scattered polarized muons off a longitudinal polarized deuteron target and detected charm mesons which are presumed to originate from the subprocess $\gamma+g \rightarrow q \bar{q}$ and are produced at high $Q^{2}$. Their result, namely that $\Delta g(x) / g(x) \sqrt{1}$ is negative for $\langle x\rangle \simeq 0.11$ [23], led to their conclusion that "This is a hint for a small value of the first moment, $\Delta G$, of the gluon helicity distribution, although this in principle does not exclude a large value." The measurements reported a year earlier at Pacific-SPIN07 of inclusive $\pi^{0}$ jets at RHIC are best fit with $\Delta G$ consistent with zero [24, 7]. Bianchi [25, 5] reported a small but non-zero $\Delta G / G \sim 0.08$ at Pacific-SPIN07 - see also the presentation by Kabuss [26] at the same conference.

A recent global analysis of parton helicity densities by deFlorian et al. [27] incorporated DIS data as well as the newly published $A_{L L}$ measurements at RHIC [28]. Based on this analysis Ref. [27] concluded that $\Delta G$ is small. However, $\Delta g(x)$ has only been measured in a limited $x$-interval, e.g., $0.06<$ $x<0.4$ in the latest RHIC measurements [28], and it is desirable to expand the measurements to a larger $x$-range before a firm conclusion on a precise value $\Delta G$ can be reached as stressed in the talks by Aidala [29] and Ellinghaus [30] at the SPIN2008 conference.

It should be noted that in recent preprints [31] Leader et al. have some critical comments on the extraction of $\Delta G$ from the earlier COMPASS Collaboration data [4]. Leader et al. find that especially the CLAS data demand higher twist (HT) terms and they state that "They [COMPASS] do not include HT terms". Ref. [31] continues to say: "We fail to find negative $\Delta g(x)$ fits without HT terms!". In their presentation at SPIN2008 they [31] stated that present day data cannot distinguish between a positive or negative value for $\Delta G$.

If as a rough estimate we assume $\Delta G \simeq 0.2$ and $\alpha_{s}\left(Q^{2}\right) \simeq 0.3$ for $Q^{2} \simeq 3$

\footnotetext{
1 The Bjorken $x$ ranges from $10^{-5}$ to 0.6 in Ref. [23].
} 
$(\mathrm{GeV} / \mathrm{c})^{2}$ we find for $N_{f}=3$ that the second term in Eq.(3) contributes about $0.03 \ll \Sigma_{\text {quark }}$. In other words, a value for $\Delta G$ much larger than 0.2 is necessary in order to reconcile the observed quark spin content of the proton with the expectations in a relativistic quark picture.

\section{The modern explanation of the value of $\Sigma$}

There were two recent developments which inspired us to re-examine the evaluation of $\Sigma$ within the successful quark model description of proton structure. First, as we already discussed, the current experimental evidence shows that polarized gluons cannot explain a major part of the observed reduction in $\Sigma$. Second, new studies in lattice QCD evaluations of the masses of the nucleon and $\Delta$ as a function of quark mass have resulted in the discovery [32, 33$]$ that the pion loops yield only $40 \pm 20 \mathrm{MeV}$ of the nucleon - $\Delta$ mass difference. These two developments led us to reconsider an explanation for the $\Sigma$ value made shortly after the original EMC results were known [34, 35, 36, 37].

In this section we will demonstrate that well known, non-perturbative QCD aspects of nucleon structure, involving its pion cloud and the quark hyperfine interaction mediated by an effective one-gluon exchange force combined with the relativistic motion of the confined quarks, not only explain the baryon magnetic moments and their semi-leptonic decays but also give a very satisfactory explanation of the modern experimental value of $\Sigma$ [38]. A consequence of this new insight is that the missing spin should be accounted for by the orbital angular momentum of the quarks and anti-quarks, a topic we will discuss further in sections four and five.

In the limit $Q^{2} \rightarrow \infty$ the Bjorken sum rule, as derived from dispersion theory [39], says that the integral over the proton spin structure function, $g_{1}^{p}(x)$, equals a low-energy axial current matrix element of the proton with $s_{z}=+1 / 2$

$$
\Gamma_{1}^{p}=\int_{0}^{1} \mathrm{~d} x g_{1}^{p}\left(x, Q^{2} \rightarrow \infty\right)=\left\langle p \uparrow\left|\sum_{i} \bar{\Psi}_{i} q_{i}^{2} \gamma_{5} \gamma_{3} \Psi_{i}\right| p \uparrow\right\rangle,
$$

where $q_{i}$ is the charge of quark $i$ (in units of the proton charge). The r.h.s. of Eq. (41) was written in Eq. (1) as the sum of an isovector, an $S U_{F}(3)$ octet and a flavor singlet component.

In the naive parton model only the one-body axial currents are considered when the matrix element in Eq.(4) is evaluated. However, the proton

contains three confined, interacting valence quarks and below we will present 
the major contributions to the axial current of the low-energy proton matrix element on the r.h.s. of Eq. (4). Specifically, the matrix element of the axial current in Eq.(4) includes contributions generated by two-quark axial operators [34] and is strongly influenced by the pion cloud [35]. In the following explanation of the measured value of $\Sigma$ we will use as guidance the results which are obtained by the cloudy bag model [40, 41], a model which successfully describes nucleon observables including the axial coupling $g_{A}-$ e.g. Ref [42].

\subsection{Relativistic valence quark}

Even at the time when the results of the EMC experiment were published it was known that the motion of the confined quarks would reduce the value of $\Sigma$. The current $u$ and $d$ quark masses are small compared to the QCD scale, $\Lambda_{Q C D} \simeq 300 \mathrm{Mev} / \mathrm{c}$, and in a space of dimension of $1 \mathrm{fm}$ the light quark moves relativistically. A spin-up quark in an s-state has a lower p-wave Dirac component. For a proton with spin up this lower component naturally has spin-down and thereby reduces the "spin content" of the valence quark. In the bag model, which is a spherical confining cavity of radius $R=1$ $\mathrm{fm}$, it is an excellent approximation to work with massless $u$ and $d$ quarks which have a minimal energy $E_{q}=\Omega / R$, where $\Omega \simeq 2.04$. The reduction factor for the axial charge of the light quarks in the bag, compared with the non-relatistic limit, is $B=\Omega / 3(\Omega-1) \simeq 0.65$. This value changes very little if we use typical light quark current masses, $m_{u} \simeq 7 \mathrm{MeV}$ and $m_{d} \simeq 15 \mathrm{MeV}$. We also note that even in modern relativistic models, where quark confinement is simulated by forbidding on-shell propagation through proper time regularization, the reduction factor is very similar. For example, Ref. [43] finds a factor 0.67 and a similar result is found in Ref. [44]. In other words, the valence quarks' orbital motion account for roughly $35 \%$ of the nucleon spin.

\subsection{The quark-quark hyperfine interaction}

It is well-established that the hyperfine interaction between quarks in a baryon, mediated by an effective one-gluon-exchange (OGE) interaction, explains the major part of the baryon octet and decuplet mass difference - e.g. the nucleon- $\Delta$ and $\Sigma-\Lambda$ mass differences [45, 46]. This spin-spin (OGE) interaction will therefore also contribute when an external probe interacts 


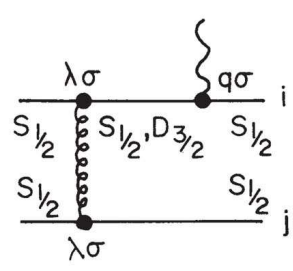

(a)

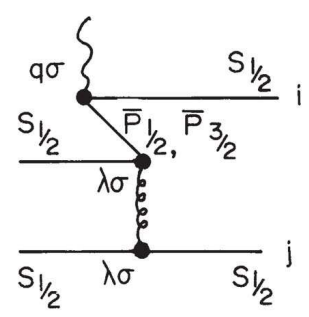

(c)

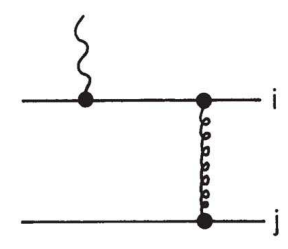

(b)

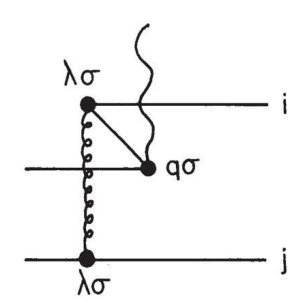

(d)

Figure 1: We illustrate the quark-quark hyperfine contributions which involve an excited intermediate quark state. In the figures the external probe (top vertical wavy line) couples to the i'th quark which interacts with the second j'th quark via the effective one gluon exchange. The intermediate quark propagator is evaluated as a sum over confined quark modes. In Figs. (a) and (b) we illustrate the three-quark intermediate states, and in (c) and (d) the one anti-quark and four quarks intermediate states. The mode sum converges rapidly and the lowest anti-quark $P_{1 / 2}$ and $P_{3 / 2}$ modes dominate the mode-sum [47]. 
with the three-quark baryon state. That is, the probe not only senses a single quark current but a two-quark current as well. This two-quark current has an intermediate quark propagator connecting the probe and the hyperfine-interaction vertices as illustrated in Fig. 1.

In the exploration of the two-quark axial current by Høgaasen and Myhrer [47], the MIT bag model was used and the confined quark propagator was written as a sum over quark eigenmodes. The dominant OGE exchange current corrections to the octet magnetic moments and semi-leptonic decays were found to come from the intermediate p-wave anti-quark states. They found that this correction was vital in reproducing not only the observed magnetic moment ratio of the two baryons $\Xi^{-}$to $\Lambda 2$ but also the unusual strength of the decay $\Sigma^{-} \rightarrow n+e^{-}+\bar{\nu}_{e}$.

Myhrer and Thomas [34] realized the importance of this OGE correction to the flavor singlet axial charge and hence to $\Sigma_{\text {quark }}$ in Eq.(11). They concluded that this OGE correction reduced the fraction of the spin carried by the quarks in the proton by 0.15 , i.e. $\Sigma_{\text {quark }} \rightarrow \Sigma_{\text {quark }}-3 G$. The correction term $G \simeq 0.05$, found by summing over the quark states, is proportional to $\alpha_{s}$ times a bag model matrix element [47] where $\alpha_{s}$ is determined by the "bare" nucleon - $\Delta$ mass difference. Again the spin is lost to angular momentum of quarks and antiquarks, the latter predominantly in p-waves.

The importance of the hyperfine interaction among quarks with respect to the large- $x$ behaviour of parton distribution functions was discussed by Close and Thomas [48] and Isgur [49].

\subsection{The pion cloud}

The virtual pion emission and absorption by the nucleon quark core is an effective implementation of the chiral symmetry requirements and is crucial in describing the charge radii of the proton and neutron, as well as other properties of the nucleon [40, 41, 42, 50]. The cloudy bag model (CBM) [40, 41] incorporates the chiral symmetry requirements and is highly successful in describing baryon properties. In this model the nucleon consists of a bare nucleon, $\mid N>$, with a probability $Z \sim 1-P_{N \pi}-P_{\Delta \pi} \sim 0.7$, in addition to being described as a nucleon $N$ and a pion and a $\Delta$ and a pion, with probabilities $P_{N \pi} \sim 0.20-0.25$ and $P_{\Delta \pi} \sim 0.05-0.10$, respectively. The phenomenological constraints on these probablilities were discussed in Refs. [51, 52, 53]. The

\footnotetext{
2 According to PDG this ratio is larger than one; (-0.6507 \pm 0.0025 n.m.) / (-0.613 \pm 0.004 n.m.). Most models without the OGE find this ratio smaller than one.
} 
most well-known of these constraints is associated with the excess of $\bar{d}$ over $\bar{u}$ quarks in the proton, predicted on the basis of the CBM [54]. Indeed, to first order the integral of $\bar{d}(x)-\bar{u}(x)$ is $2 / 3 P_{N \pi}-P_{\Delta \pi} / 3$, which is consistent with the experimental data [55] if $P_{N \pi}$ and $P_{\Delta \pi}$ lie within the ranges just quoted.

The effect of the pion cloud on the quark spin contribution was investigated early by Schreiber and Thomas [35. They wrote the corrections to the spin sum-rules for the proton and neutron explicitly in terms of the probabilities set out above. For the present purposes it is helpful to rewrite the results of Ref. [35]. If we consider the flavor singlet combination the pion cloud correction modifies the quark spin contribution in the following manner:

$$
\Sigma_{\text {quark }} \rightarrow\left(Z-\frac{1}{3} P_{N \pi}+\frac{5}{3} P_{\Delta \pi}\right) \Sigma_{\text {quark }}
$$

The critical feature of the pion cloud correction in Eq. (5) is that the ClebschGordon algebra for coupling the spin of the proton and the orbital angular momentum of the pion in the $N \pi$ Fock state favors a spin down nucleon and a pion with +1 unit of orbital angular momentum. This too has the effect of replacing quark spin by quark and anti-quark orbital angular momentum. Note that in the $\Delta \pi$ Fock component the spin of the baryon tends to point up (and the pion angular momentum down), thus enhancing the quark spin. Nevertheless, the wave function renormalization factor, $Z$, dominates, yielding a reduction by a factor between 0.7 and 0.8 for the range of probabilities quoted above.

\subsection{The possible polarized gluon contribution $\Delta G$}

We have used a model of confined quarks to compute the matrix elements of the axial current to find $\Sigma$ and $g_{A}$ values relevant in the limit $Q^{2} \rightarrow$ $\infty$. Our model result, $\Sigma_{\text {quark }} \in(0.35,0.40)$ [38], agrees very well with the experimental value $\Sigma$ - c.f. Eq.(2). Although there is no unambiguous way to identify the scale associated with the chiral quark model, many authors (see e.g., Refs. [56, 57, 58]) have made the observation that a valence dominated quark model can only match experiment for parton distribution functions at a relatively low $Q^{2}$ scale. We argue that the quark model evaluation of the proton matrix element of the flavor singlet axial current corresponds to the first term in Eq.(3). To explain the reasoning in more detail we observe the following. In the limit $Q^{2} \rightarrow \infty$ the product $\alpha_{s}\left(Q^{2}\right) \Delta G\left(Q^{2}\right)$ in Eq.(3) 
tends to a constant [17]. In the same $Q^{2}$ limit the flavor singlet part of the $x$ integral over $g_{1}\left(x, Q^{2}\right)$ of Eq.(1) equals the low-energy proton matrix element of the singlet axial current

$$
\sum_{i=u, d, s}<p\left|\bar{q}_{i} \gamma^{\mu} \gamma_{5} q_{i}\right| p>
$$

which contains a contribution from the quarks as well as the axial anomaly term involving $\alpha_{s}\left(Q^{2}\right) \Delta G\left(Q^{2}\right)$. The separation of the two contributions in Eq.(3) is most naturally made in terms of the range of integration over the transverse quark momenta, $k_{\perp}^{2}$. The contribution of the axial anomaly in Eq.(3) relies on the scale separations [17]: $m_{\text {quark }}^{2} \ll\left(1 / R_{\text {confinement }}^{2}\right) \sim$ $p_{\text {gluon }}^{2} \ll Q^{2}$, and the integration over transverse momenta is dominated by the range $k_{\perp}^{2} \geq Q^{2}$ [17, 20]. For comparison, the contribution from valence quarks is dominated by relatively low transverse momenta, $k_{\perp}^{2}<$ $1(\mathrm{GeV} / \mathrm{c})^{2}<Q^{2}$. The valence parton distributions calculated in this way correspond to the original Gribov-Lipatov definition of a parton distribution at scale $Q^{2}$. Numerically, we note that if the value of $\Delta G \simeq 0.4$ at $Q^{2} \simeq 3(\mathrm{GeV} / \mathrm{c})^{2}$ as inferred from recent results at RHIC [59], then the second term in Eq.(3) is estimated to contribute about 0.06 to $\Sigma$, using $\alpha_{s}\left(Q^{2}\right) \simeq 0.3$ at this $Q^{2}$ scale.

\section{Lattice QCD calculations of quark orbital angular momentum}

The results of the last section imply that a large fraction of the proton spin is carried by quark and anti-quark orbital angular momenta. The distributions of the orbital angular momenta for $u+\bar{u}$ and $d+\bar{d}$ were extracted from our model results by Thomas [60] and he found that $L^{u+\bar{u}} \simeq .50$ and $L^{d+\bar{d}} \simeq 0.12$, which give $J^{u+\bar{u}}=L^{u+\bar{u}}+\Delta u / 2 \simeq 0.72$ and $J^{d+\bar{d}}=$ $L^{d+\bar{d}}+\Delta d / 2 \simeq-0.10$. These values should be compared to the QCD lattice results for orbital angular momentum as evaluated by the LHPC collaboration [61]. The lattice QCD $L^{u+\bar{u}}$ and $L^{d+\bar{d}}$ values were however evaluated at a scale of about $4 \mathrm{GeV}^{2}$, much larger than the scale relevant for the chiral quark model. In order to compare the angular momentum results of the model with the lattice QCD results, Thomas [60] used QCD evolution equations for angular momentum as outlined in the work by Ji and collaborators [62. 3 Thomas showed that the QCD evolution has a dramatic influence

\footnotetext{
3 Ji 63] introduced a gauge-invariant decomposition of the nucleon spin into quark helicity, quark orbital and gluon contributions. Recently there has been some debate on the proper way to define the quark orbital angular momentum, see e.g. Refs. 64, 65, 66, 67, 68].
} 
on the different quarks' orbital angular momenta as a function of $Q^{2}$ and that the model results, which are derived based on the results of the previous section, are consistent with the lattice QCD results and also with results for $J^{u+\bar{u}}$ and $J^{d+\bar{d}}$, which have been extracted from DVCS experiments at DESY [69] and JLab [70] using the model of Goeke et al. [71] - see also [72].

Although the predictions of the Myhrer-Thomas (MT) work are consistent with both the lattice QCD data and the model dependent analysis of recent experiments, it is important to realize that at present this is not a compelling statement. The nature of the QCD evolution is such that predictions which differ dramatically at the model scale tend to be much closer at $4 \mathrm{GeV}^{2}$. For example, a change in the MT value for $L^{u}-L^{d}$ by 0.10 at the model scale leads to a change of only 0.01 at $4 \mathrm{GeV}^{2}$. Clearly, this makes the challenge of making measurements, either experimentally or on the lattice, which clearly discriminate between models, very difficult indeed. Furthermore, we should note that the current lattice calculations suffer from the omission of "disconnected quark loops", which contain the effect of the axial anomaly and this introduces a completely unknown systematic error quite apart from the usual uncertainties of taking the chiral limit, the lattice spacing to zero and the volume to infinity.

It is also very important to note that, as pointed out by Wakamatsu and Tsujimoto, whereas the chiral quark soliton model usually yields very similar results to those found in the CBM, in the case of $L^{u}-L^{d}$ they are completely different [73]. Indeed, the non-linear pion fields in the chiral quark soliton model yield a large negative prediction for this quantity at the model scale. For the moment the uncertainties that we summarised in the previous paragraph make discrimination between the models on this basis impossible at present but this may prove a critical discriminator as the lattice computations improve over the next few years.

\section{The GPDF and quark angular momentum}

Some time ago the transverse quark degrees of freedom in hadrons were explored in order to gain some understanding of the high $Q^{2}$ large scattering angle behavior of $p p$ cross section, analyzing power and $A_{N N}$. In these large $Q^{2}$ measurements the short-range ("hard") scattering processes dominate. However, the transverse hadronic dimensions, which are determined by confinement and generate "medium -range" interactions among the valence quarks, can interfere and successfully explain these $Q^{2}$ phenomena [74, 75]. 
In addition, these medium-range interactions gave some possible insight as to why the measured exclusive cross sections not only exhibit the expected hard-scattering $Q^{2}$ scaling behavior but also explain the superimposed oscillatory behavior of the cross sections versus $Q^{2}$ (for a fixed large scattering angle) in measured exclusive hadronic reactions, e.g. pp and $\pi$ p scattering. The inferred conclusion was that even if helicity is conserved in the perturbative quark-gluon processes, helicity is not necessarily conserved at the hadronic level. In short, the transverse components of a hadron introduce quark angular momentum and possible quark spin-orbit interactions into the description of hadronic spin high $Q^{2}$ observables at large scattering angles.

To examine the importance of the quark orbital angular momentum one has to study the Generalized Parton Correlation Functions (GPCF) which could be extracted from measurements. A concise overview of possible measurements of GPCF and their connection to the quarks transverse degrees of freedom, has been presented in a recent COMPASS report [76]. A detailed exposition of recently proposed non-trivial relations between generalized and transverse momentum dependent parton distributions (GPD and TMD, respectively) are given in Ref. [77]. According to Ref. [77] there are no modelindependent relations between GPD and TMD functions. It is unfortunate that model considerations are necessary in order to extract the transverse momentum dependences of GPCF from measured observables. Recently Efremov et al. [78] used a covariant model of free quarks to discuss relations between GPDs and TMDs which can be derived from GPCF. Some of these relations have also been derived in several other parton model studies, see e.g., Ref. [78] and references therein. As argued by Burkardt [79], parton distributions in impact parameter space show significant deviation from axial symmetry when, for example, the proton is transversely polarized. Extracting this axial asymmetry using, for example, the Sivers function $f_{1 T}^{\perp q}\left(x, Q^{2}\right)$ could provide useful information about quark angular momentum, however as mentioned one has to resort to a model to extract the necessary information. Ma et al. [80] showed that pretzelosity, another TMD, could give useful information about the quark angular momentum but a quark-diquark model is necessary to extract this information. A recent model discussion on pretzelosity can be found in Avakian et al. [81]. According to Meissner et al. [77] the TMDs are determined when hadron momentum transfer $\Delta=p^{\prime}-p=0$ after an integration over $k^{-}$of the GPCFs. In the forward limit, $\Delta \simeq 0$, a word of caution has been advocated by Szczepaniak et al. [82, 83] who show 
that it is a non-trivial task to extract TMDs from measured data. They also present evidence for a fixed $J=0$ pole contribution to deep virtual Compton scattering at all momentum transfers. This acts like a subtraction in the dispersion relation for the Compton amplitude [84, 83].

\section{Concluding remarks}

We have seen that the latest data on the proton spin sum-rule, which yields the result that the fraction of the spin of the proton carried by its quarks, $\Sigma=33 \pm 3 \pm 5 \%$, is very naturally explained within a relativistic quark model that includes the effective one-gluon-exchange hyperfine interaction and respects chiral symmetry. In many ways these are what might be regarded as the basic ingredients of a modern model of nucleon structure and it is very satisfying that the proton spin crisis, which has caused such consternation in the nuclear and particle physics communities over the past 20 years, can be explained this way.

The role of the axial anomaly is now known to be considerably smaller than was once hoped, with $\Delta G$ now most likely between 0 and 0.4 [59] at $Q^{2}$ of order $4 \mathrm{GeV}^{2}$. Nevertheless, even such a small contribution will become significant as the precision with which $\Sigma$ is determined increases. Indeed, the corresponding contribution to $\Sigma$ from gluons in this range would be between 0.06 and 0 . As a consequence of this, the gauge invariant quark spins, $\Delta u, \Delta d$ and $\Delta s$ would each receive a gluonic contribution as large as -0.02. A simple kaon loop calculation suggests a chiral contribution to $\Delta s$ of order -0.01 , resulting in a total value of $\Delta s$ as large as -0.03. Testing this directly, for example through neutral current neutrino-proton elastic scattering, would be extremely valuable.

It is interesting to note the upper limit on the magnitude of $\Delta s$ derived in this way, namely $\Delta s=-0.03$, is considerably lower than the value derived from $\Sigma=\Delta u+\Delta d+\Delta s$ (given above) and the value of $g_{A}^{8}=\Delta u+\Delta d-$ $2 \Delta s=0.57 \pm 0.03$ usually derived from hyperon $\beta$-decay, namely $\Delta s=$ $-0.08 \pm 0.01 \pm 0.02$. Of course, it has been argued [10] that the error in applying $\mathrm{SU}(3)$ symmetry to the octet axial charges may be as large as $20 \%$, which could bring the value of $\Delta s$ derived from $\Sigma$ and $g_{A}^{8}$ as low as $-0.04 \pm 0.01 \pm 0.02$, which would be in much better agreement. It is clearly important to carefully derive theoretical values for both $\Sigma$ and $g_{A}^{8}$ within any model applied to the spin problem.

While Occham's razor suggests that the simplest explanation is almost certainly the correct one, we note that there are several other proposals which 
are also able to explain the experimental result. Using a generalization of the Goldberger-Treiman relation to the singlet case, Shore and Veneziano [85, 86, 87] predicted a reduction of about $50 \%$ in the naive relativistic expectation for $\Sigma$. This model has interesting predictions for the corresponding suppression in other hadrons.

Another fascinating suggestion from Bass [21, 88, 89] sees much of the spin of the proton tied up in the topological structure of the gluon fields. This would show up only through a $J=1$ fixed pole which contributes to the structure functions as a $\delta$-function at $x=0$. One signature of such an effect would be a difference in the value of $\Sigma$ extracted from neutrino-proton elastic scattering from that obtained in deep inelastic scattering - where the $\delta$-function at $x=0$ is unmeasureable.

Finally, we note that while the gross violation of quark model expectations has now been removed, so that in our view the "spin crisis" has been solved, the problem of understanding in detail how the spin of the proton is carried by its quarks and gluons is now of great interest. On the scale of one half, it matters a great deal whether $\Delta G$ is 0 or 0.4 . The Myhrer-Thomas explanation of the spin crisis implies that much of the proton spin is carried as orbital angular momentum and it is critical to find ways to pin this down. We have seen that the study of GPDs through both lattice QCD and experiment will be crucial in this quest and the $12 \mathrm{GeV}$ upgrade at Jefferson Lab [90] is ideally suited to play a key role, at least in the valence region. For the sea-quark region we may well need a high luminosity electron-ion collider [91] but that may take a little longer.

Acknowledgements This work was supported by the US Department Energy, Office of Nuclear Physics, through contract no. DE-AC05-06OR23177, under which Jefferson Science Associates operates Jefferson Lab and by the NSF grant PHY-0758114.

\section{References}

[1] J. Ashman et al. [European Muon Collaboration], Phys. Lett. B 206, 364 (1988).

[2] B. Adeva et al.[SMC Collaboration], Phys. Rev. D 58, 112001 (1998)

[3] E. S. Ageev et al. [COMPASS Collaboration], "Phys. Lett. B 612, 154 (2005) arXiv:hep-ex/0501073. 
[4] V.Y. Alexakhin et al. [COMPASS Collaboration], Phys. Lett. B 647, 8 (2007) [arXiv:hep-ex 0609.038]

[5] A. Airapetian et al. [HERMES Collaboration], Phys. Rev. D 75, 012007 (2007) [arXiv:hep-ex 0609.039]; ibid. 76, 039901 (2007), Erratum.

[6] K.V. Dharmawardane et al.[CLAS Collaboration], Phys. Lett. B 641, $11(2006)$.

[7] B. I. Abelev et al. [STAR Collaboration], Phys. Rev. Lett. 97, 252001 (2006); ibid. 100, 232003 (2008).

[8] A. Adare et al. [PHENIX Collaboration], Phys. Rev. D 76, 051106 (2007).

[9] V.W. Hughes, Nucl. Phys. A 518, 371 (1990), and references therein.

[10] R. L. Jaffe and A. Manohar, Nucl. Phys. B 337, 509 (1990).

[11] S. A. Larin and J. A. M. Vermaseren, Phys. Lett. B 259, 345 (1991).

[12] S. A. Larin, Phys. Lett. B 334, 192 (1994) arXiv:hep-ph/9403383; S. A. Larin, T. van Ritbergen and J. A. M. Vermaseren, Phys. Lett. B 404, 153 (1997) arXiv:hep-ph/9702435.

[13] S.E. Kuhn, J.-P. Chen and E. Leader, Prog. Nucl. Part. Phys. 63, 1 (2009).

[14] S.D. Bass, arXiv(hep-ph):0905.4619

[15] A. V. Efremov and O. V. Teryaev, Report JINR-E2-88-287 (1988).

[16] G. Altarelli and G. G. Ross, Phys. Lett. B 212, 391 (1988).

[17] R.D. Carlitz, J.C. Collins and A.H. Mueller, Phys. Lett. B 214, 229 (1988)

[18] E. Leader and M. Anselmino, Z. Phys. C 41, 239 (1988); AIP Conf. Proc. 187, 764 (1989).

[19] G. T. Bodwin and J. W. Qiu, Phys. Rev. D 41, 2755 (1990). 
[20] S. D. Bass, B. L. Ioffe, N. N. Nikolaev and A. W. Thomas, J. Moscow. Phys. Soc. 1, 317 (1991).

[21] S. D. Bass, Rev. Mod. Phys. 77, 1257 (2005) arXiv:hep-ph/0411005].

[22] E. Rondio, plenary talk (Oct. 10) at the 18th Intern. Symp. on Spin Physics (SPIN2008), Charlottesville, VA, Oct. 9-11, 2008. http://faculty.virginia.edu/spin2008/scientific_ program.html

[23] M. Alekseev et al. [COMPASS Collaboration], Phys. Lett. B 676, 31 (2009)

[24] A. Bazilevsky [PHENIX Collaboration], invited talk presented at Pacific-SPIN07, July 30-August 2, 2007 http://www.triumf.info/hosted/pacspin07/program.htm

[25] N. Bianchi [HERMES Collaboration], invited talk presented at PacificSPIN07, July 30-August 2, 2007 (op. cit.).

[26] E.-M. Kabuss [COMPASS Collaboration], invited talk presented at Pacific-SPIN07, Vancouver, Canada.

[27] D. de Florian, R. Sassot, M. Stratmann, and W. Vogelsang, Phys. Rev. Lett. 101, 072001 (2008)

[28] A. Adare et al. [PHENIX Collaboration], Phys. Rev. D 79, 012003 (2009).

[29] C. Aidala, plenary talk (Oct. 9) at the 18th Intern. Symp. on Spin Physics (SPIN2008), Charlottesville, VA, Oct. 9-11, 2008. http://faculty.virginia.edu/spin2008/scientific program.html; $\operatorname{arXiv}($ hep-ex):0903.2393 (2009)

[30] F. Ellinghaus, talk (Oct. 6) at the 18th Intern. Symp. on Spin Physics (SPIN2008), Charlottesville, VA, Oct. 9-11, 2008. http://faculty.virginia.edu/spin2008/scientific_ program.html

[31] E. Leader, A.V. Sidorov and D.B. Stamenov, "Status of Longitudinal Polarized Parton Densities and Higher Twist", to appear in Proc. 18th Intern. Symp. on Spin Physics (SPIN2008), arXiv[hep-ph]:0901.2285 (2009); "Gluon Polarization and Higher Twist Effects" (Proceedings 
of DIS-2008) arXiv:[hep-ph]0806.2094 (2008); Phys. Rev. D 75, 074027 (2007)

[32] R. D. Young, D. B. Leinweber, A. W. Thomas and S. V. Wright, Phys. Rev. D 66, 094507 (2002) arXiv:hep-lat/0205017.

[33] R. D. Young, D. B. Leinweber, A. W. Thomas and S. V. Wright, Nucl. Phys. B ( Proc. Suppl. ) 109 (1), 55 (2002).

[34] F. Myhrer and A. W. Thomas, Phys. Rev. D 38, 1633 (1988).

[35] A. W. Schreiber and A. W. Thomas, Phys. Lett. B 215, 141 (1988).

[36] H. Høgaasen and F. Myhrer, Phys. Lett. B 214, 123 (1988).

[37] K. Kubodera, Y. Kohyama, K. Tsushima and T. Yamaguchi, Proc. XXIII Yamada Conf. on Nuclear Weak Process and Nuclear Structure, June 1989, Osaka, Japan, eds. M. Morita, H. Ejiri, H. Ohtsubo and T. Sato (World Scientific, Singapore 1989), p.408.

[38] F. Myhrer and A.W. Thomas, Phys. Lett. B 663, 302 (2008).

[39] J. Bjorken, Phys. Rev. 148, 1467 (1966)

[40] S. Theberge, A. W. Thomas and G. A. Miller, Phys. Rev. D 22, 2838 (1980) [Erratum-ibid. D 23, 2106 (1981)].

[41] A. W. Thomas, Adv. Nucl. Phys. 13, 1 (1984).

[42] K. Tsushima, T. Yamaguchi, Y. Kohyama and K, Kubodera, Nucl. Phys. A 489, 557 (1988).

[43] I. C. Cloet, W. Bentz and A. W. Thomas, Phys. Lett. B 659 (2008) 214 arXiv:0708.3246 [hep-ph].

[44] D. Qing, X.-S. Chen and F. Wang, Phys. Rev. D 58, 114032 (1998).

[45] A. De Rujula, H. Georgi and S. L. Glashow, Phys. Rev. D 12, 147 (1975).

[46] A. Chodos, R. L. Jaffe, K. Johnson and C. B. Thorn, Phys. Rev. D 10, 2599 (1974). 
[47] H. Høgaasen and F. Myhrer, Phys. Rev. D 37, 1950 (1988)

[48] F. E. Close and A. W. Thomas, Phys. Lett. B 212, 227 (1988).

[49] N. Isgur, Phys. Rev. D 59, 034013 (1999)

[50] F. Myhrer, G.E. Brown and Z. Xu, Nucl. Phys. A 362, 317 (1981).

[51] J. Speth and A. W. Thomas, Adv. Nucl. Phys. 24, 83 (1997).

[52] W. Melnitchouk, J. Speth and A. W. Thomas, Phys. Rev. D 59, 014033 (1999) arXiv:hep-ph/9806255].

[53] A.W. Thomas, Prog. Theor. Phys. Suppl., 168, 614 (2007); [arxiv:nuclth/0711.2259]

[54] A. W. Thomas, Phys. Lett. B 126, 97 (1983).

[55] M. Arneodo et al. [New Muon Collaboration], Nucl. Phys. B 487, 3 (1997) arXiv:hep-ex/9611022].

[56] G. Parisi, Phys. Lett. B 43, 207 (1973).

[57] A. I. Signal and A. W. Thomas, Phys. Lett. B 211, 481 (1988).

[58] M. Gluck and E. Reya, Z. Phys. C 43, 679 (1989).

[59] A. Adare et al. [PHENIX Collaboration], Phys. Rev. Lett. 103, 012003 (2009) arXiv:0810.0694 [hep-ex]].

[60] A.W. Thomas, Phys. Rev. Lett. 101, 102003 (2008); [arXiv (hep-ph): 0803.2775]

[61] Ph.Hagler et al. [LHPC Collaboration], Phys. Rev. D 77, 094502 (2008); D. Richards, arXiv:0711.2048.

[62] X.D. Ji, J. Tang and P. Hoodbhoy, Phys. Rev. Lett. 76, 740 (1996).

[63] X.D. Ji, Phys. Rev. Lett. 78, 610 (1997).

[64] X. S. Chen, X. F. Lu, W. M. Sun, F. Wang and T. Goldman, Phys. Rev. Lett. 100, 232002 (2008) arXiv:0806.3166 [hep-ph]].

[65] X. Ji, arXiv:0810.4913 [hep-ph]. 
[66] X. S. Chen, X. F. Lu, W. M. Sun, F. Wang and T. Goldman, arXiv:0812.4336 [hep-ph].

[67] X. S. Chen, W. M. Sun, X. F. Lu, F. Wang and T. Goldman, Phys. Rev. Lett. 103, 062001 (2009) [arXiv:0904.0321 [hep-ph]].

[68] M. Burkardt and Hikmat BC, arXiv:0812.1605 [hep-ph].

[69] F. Ellinghaus et al., Eur. Phys. J. C 46, 729 (2006); Z. Ye [HERMES Collaboration], arXiv:hep-ex/0606061

[70] M. Mazouz et al. [Jefferson Lab Hall A Collaboration], Phys. Rev. Lett. 99, $242501(2007)$

[71] K. Goeke et al., Prog. Part. Nucl. Phys.47, 401 (2001).

[72] M. Vanderhaeghen, P.A.M. Guichon and M. Guidal, Phys. Rev. D 60, 094017 (1999).

[73] M. Wakamatsu and H. Tsujimoto, Phys. Rev. D 71, 074001 (2005) arXiv:hep-ph/0502030].

[74] J.P. Ralston and B. Pire, Phys. Rev. Lett. 57, 2330 (1986).

[75] C.E. Carlson, M. Chachkhunashvili and F. Myhrer, Phys. Rev. D 46 , 2891 (1992); F. Myhrer, Phys. At. Nucl., 57, 1559 (1994).

[76] COMPASS collab., preprint CERN-SPSC-2009-003/SPSC-I-238.

Preprint sent us by Steven Bass a long time ago.

[77] S. Meissner, A. Metz and M. Schlegel, arXiv(hep-ph):0906.5323 (2009)

[78] A.V. Efremov, P. Schweitzer, O.V. Teryaev and P. Závada, arXiv[hepph]:0903.3490 (2009); P. Závada, Eur. Phys. J. C 52, 121 (2007)

[79] M. Burkardt, arXiv[hep-ph]:0907.0296 (2009), and references therein.

[80] J. She, J. Zhu and B.-Q. Ma, "Pretzelosity $h_{1 T}^{\perp}$ and quark angular momentum, arXiv[hep-ph]:0902.3718 (2009); B.-Q. Ma and I. Schmidt, Phys. Rev. D 58, 096008 (1998) 
[81] H. Avakian, A. V. Efremov, P. Schweitzer and F. Yuan, Talk at Transversity 2008: 2nd International Workshop on Transverse Polarization Phenomena in Hard Processes, Ferrara, Italy, 28-31 May 2008; JLAB-PHY-08-875, Aug 2008. 8pp.; arXiv:0808.3982 [hep-ph].

[82] A. P. Szczepaniak and J. T. Londergan, Phys. Lett. B 643, 17 (2006) arXiv:hep-ph/0604266 ; A. P. Szczepaniak, J. T. Londergan and F. J. Llanes-Estrada, Acta Phys. Polon. B 40, 2193 (2009) arXiv:0707.1239 [hep-ph]] ;

[83] S. J. Brodsky, F. J. Llanes-Estrada, J. T. Londergan and A. P. Szczepaniak, Talk given at 17th International Workshop on Deep-Inelastic Scattering and Related Subjects (DIS 2009), Madrid, Spain, 26-30 Apr 2009. arXiv:0906.5515 [hep-ph].

[84] S. J. Brodsky, F. J. Llanes-Estrada and A. P. Szczepaniak, Phys. Rev. D 79, 033012 (2009) [arXiv:0812.0395 [hep-ph]].

[85] G. M. Shore and G. Veneziano, Phys. Lett. B 244, 75 (1990).

[86] S. Narison, G. M. Shore and G. Veneziano, Nucl. Phys. B 433, 209 (1995) arXiv:hep-ph/9404277.

[87] S. Narison, G. M. Shore and G. Veneziano, Nucl. Phys. B 546, 235 (1999) arXiv:hep-ph/9812333.

[88] S. D. Bass, Acta Phys. Polon. B 34, 5893 (2003) arXiv:hep-ph/0311174.

[89] S. D. Bass, Czech. J. Phys. 50, 109 (2000) [Czech. J. Phys. 50S1, 109 (2000)].

[90] A. W. Thomas, Eur. Phys. J. ST 140 (2007) 117.

[91] A. W. Thomas, arXiv:0907.4785 [hep-ex]. 